NASZA DERMATOLOGIA Online OUR DERMATOLOGY Online

Source of Support: Georgia Dermatopathology Associates, Atlanta, Georgia, USA.

Competing Interests: None

\section{VIMENTIN MAY REFLECT AREAS OF PATHOLOGIC INVOLVEMENT IN BIOPSIES FROM PATIENTS WITH AUTOIMMUNE SKIN DISEASES}

\author{
Ana Maria Abreu Velez ${ }^{1,3}$, Daniel Alberto Vásquez Hincapié ${ }^{2}$, \\ Michael S. Howard ${ }^{1}$ \\ ${ }^{1}$ Georgia Dermatopathology Associates, Atlanta, Georgia, USA \\ ${ }^{2}$ PECET, University of Antioquia, Medellin, Colombia, South America \\ ${ }^{3}$ Department of Dermatology, School of Medicine, University of Antioquia, Medellin, \\ Colombia, South America
}

Corresponding author: Ana Maria Abreu Velez, M.D., Ph.D.

\begin{abstract}
Introduction: Autoimmune bullous skin diseases (ABDs) represent a group of disorders of the skin and mucosa commonly associated with deposits of immunoglobulins, complement and fibrinogen, and usually directed against distinct adhesion molecules. After studing these diseases for many years, we noted alterations not only between the cells junctions of the epidermis and/or the dermal/epidermal junction, but also in dermal skin appendageal structures and in mesenchymal tissue around the blisters. Based on our findings, we wanted to determine if the observed patterns of autoimmunity correlated with cutaneous vimentin expression.

Materials and Methods: Archival biopsies previously diagnosed with ABDs by clinical, hematoxylin and eosin (H\&E) and direct and/or immunofluorescence data were stained with antibodies directed against vimentin via immunohistochemistry (IHC). We tested 30 patients affected by endemic pemphigus, 30 controls from the endemic area, and 15 normal controls. We also tested 30 biopsies from patients with bullous pemphigoid (BP), 20 with pemphigus vulgaris (PV), 8 with pemphigus foliaceus, 14 with dermatitis herpetiformis (DH) and 3 with Senear-Usher syndrome.

Results: The H\&E, DIF and vimentin patterns of positivity in the different ABDs confirmed that vimentin was compartmentalized around areas of dermal inflammation, around skin appendages and in epidermal, dermal and mesenchymal cell junction areas.

Conclusion: Vimentin may be a useful tool for highlighting patterns of microenvironmental tissue alteration in multiple ABDs. The vimentin staining pattern observed was analogous to that we have previously described for proteases and protease inhibitors in patients affected by ABDs, expanding the concept that the autoimmune process extends beyond cell junctions.
\end{abstract}

Key words: Autoimmune blistering skin diseases; vimentin; mesenchymal tissue

Abbreviations: Autoimmune bullous diseases (ABDs), bullous pemphigoid (BP), pemphigus vulgaris (PV), pemphigus foliaceus (PF), dermatitis herpetiformis (DH), endemic pemphigus foliaceus (EPF), linear IgA disease (LAD), immunohistochemistry (IHC), direct and indirect immunofluorescence (DIF, IIF), hematoxylin and eosin (H\&E), basement membrane zone (BMZ), intercellular staining between keratinocytes (ICS), intermediate filament (IF), epithelial-to-mesenchymal transition (EMT).

\section{Introduction}

Vimentin is a marker of migrating cells, and is a protein that in humans is encoded by the VIM gene [1-4]. Vimentin is a type III intermediate filament (IF) protein that is expressed in mesenchymal cells [1-4]. Current studies have revealed novel functions for vimentin related to cell migration, such as determination of cellular polarity, regulation of cell contact formation, organization and transport of signal proteins involved in cell motility [1-4]. Pemphigus and bullous pemphigoid are autoimmune bullous diseases of the skin. Pemphigus, an intraepidermal blistering disease, is categorized by autoantibodies reactive to antigens located in epidermal intercellular spaces, or on the surfaces of epidermal cells [5]. These antibodies, which have recently been shown to trigger complement activation, seem to be the source of acantholysis, the basic pathologic process of pemphigus [5-10]. 
Bullous pemphigoid (BP), an autoimmune subepidermal blistering skin disease, presents with tense blisters with or without concomitant erythema. Bullous pemphigoid blistering occurs along the lamina lucida, due to immunoglobulin $\mathrm{G}$ and/or complement deposits at the basement membrane zone (BMZ). Circulating autoantibodies are often also present, directed against hemidesmosomal molecules [5-10].

\section{Material and Methods}

In our IHC staining, we utilized monoclonal mouse antiVimentin antibody, Dako Clone V9, and stained as previously described [10-13]. Vimentin is a $57 \mathrm{kDa}$ intermediate filament protein which forms part of the cytoskeleton of vertebrate cells, and is characteristically found in cells of mesenchymal origin. Our archival patient tissue selected for this study was prepared as previously described [8-13]. Specifically, we tested 30 patients affected by endemic pemphigus, 30 controls from the endemic area, and 15 normal controls. We also tested 30 biopsies from patients with bullous pemphigoid (BP), 20 with pemphigus vulgaris $(\mathrm{PV}), 8$ with pemphigus foliaceus and 14 with dermatitis herpetiformis $(\mathrm{DH})$.

\section{Results}

Vimentin was present in all of the ABDs in areas of inflammation. Specifically, we noted positivity around most skin appendages, around disease blisters, and in dermal areas that demonstrate a high density of epithelial-to-mesenchymal tissue transitions(such as junctions between dermal blood vessels and the extracellular matrix). In these areas, increased cytokine signaling of local inflammation is present. These findings were observed in all the ABDs. In Figures 1 and 2, we demonstrate examples of this immune compartmentalization within dermal, epidermal and blister microenvironments in the ABDs. Moreover, it seems that in addition to the classic immunoglobulin and complement linear deposits at the BMZs, additional, orchestrated immunologic reorganization of the dermis is also present in these areas. Such an immunologic reorganization of the dermis could play a significant role in the pathophysiology of these disorders.

In the majority of the patients with $\mathrm{DH}$, the vimentin positivity was noted in the dermal papillae surrounding and inside the blisters, and in the dermis under the blisters. Positivity was also seen in some dermal fibroblast-like cells, and along neurovascular supplies of skin appendageal structures. In most patients with BP, linear staining was noted under the blisters. Staining was also noted in the upper and intermediate dermis, on several dermal fibroblast-like cells and around eccrine sweat ducts and blood vessels. In most of the El Bagre-EPF and the PF cases, an epidermal ICS-like pattern was seen. In addition, dermal staining was noted in the dermis under the blisters, on eccrine sweat glands, on neurovascular supplies of appendageal structures (including sebaceous glands and hair follicles), and in the subcutaneous adipose tissue. In the bulk of the PV cases, vimentin was positive in the debris within the blisters. However, the majority of the staining was found in a band-like distribution in the upper dermis, including around superficial neurovascular packages and around some skin appendageal structures. In our 3 cases of Senear-Usher syndrome, vimentin staining was primarily positive around hair follicle basal layers and around the neurovascular supplies for hair follicles and sebaceous glands.

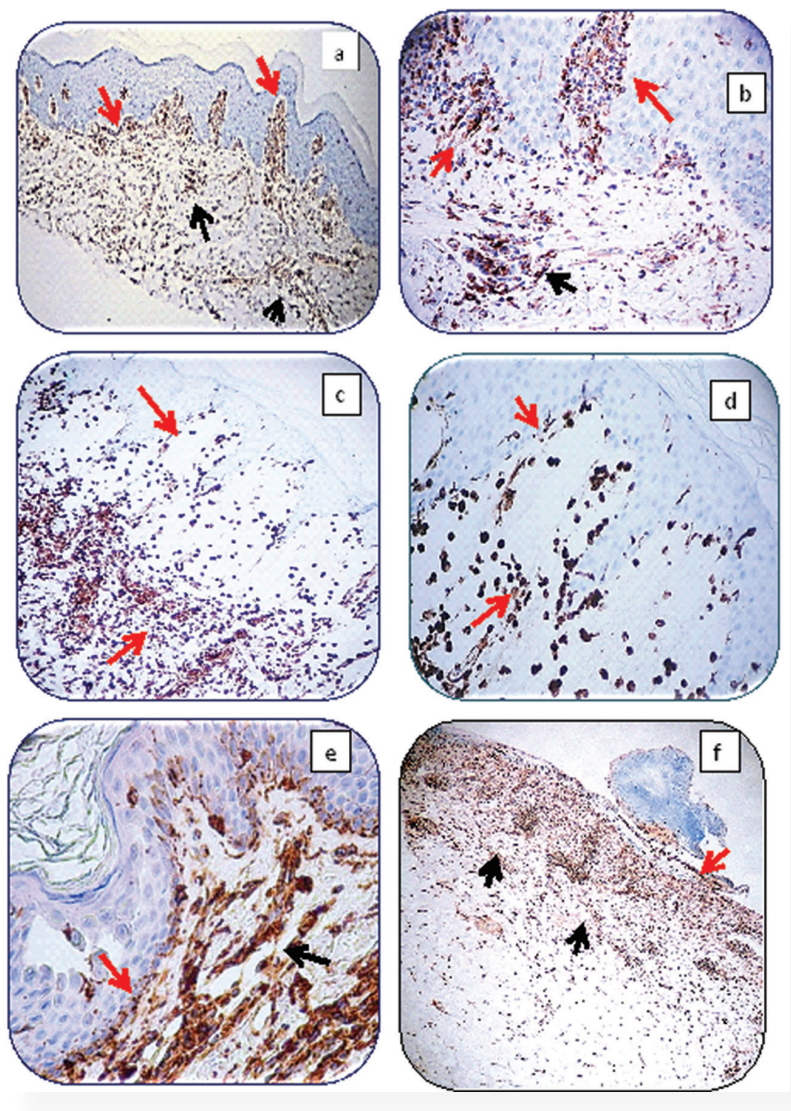

Figure 1. a. IHC staining with vimentin in $\mathrm{DH}(100 \mathrm{x})$ showing positivity around the blister in the high papillary dermis and BMZ areas (brown staining; red arrows), as well as around upper dermal blood vessels and eccrine ducts (brown staining; black arrows). b. A second DH case, demonstrating positive staining with vimentin in the dermal papillae and subepidermal blisters (brown staining; red arrows) and around upper dermal blood vessels (brown staining; black arrow)(200x). c and d. In c, a DH case, showing detailed staining with vimentin around a subepidermal blister (brown staining; red arrows) and in $\mathrm{d}$, around dermal blood vessels (brown staining; red arrows)(100 and 200x, respectively). e. Tissue inhibitor of metalloproteinase 1 staining (red staining) in a PV case, showing some positive staining in areas where vimentin staining is also positive (brown staining) (red and black arrows). f. IHC staining with vimentin (brown staining) in a PV case. The red arrow designates staining within the blister, and the black arrow highlights staining in the upper dermal tissues including around blood vessels. 

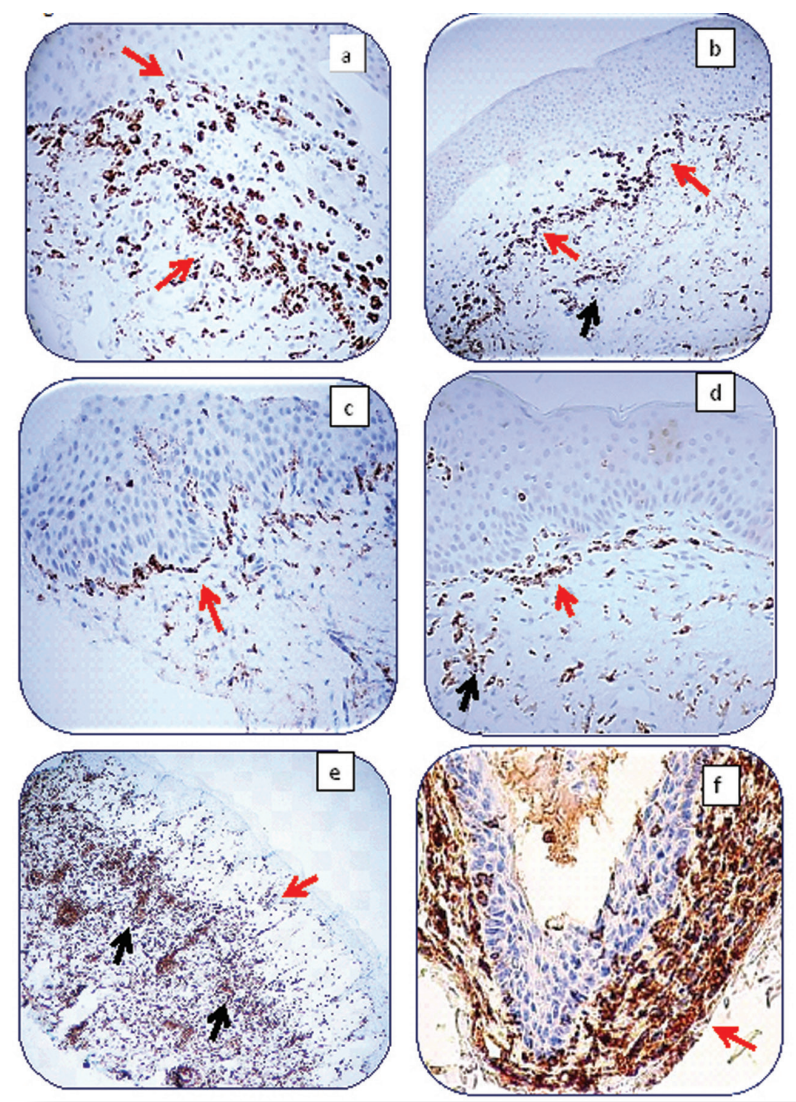

Figure 2. a through $\mathrm{d}$. IHC staining with vimentin in four different cases of $\mathrm{BP}$, showing the stain demarcating the blisters and/or linear staining at the BMZ (brown staining; red arrows). In addition, vimentin staining is noted around upper dermal blood vessels (brown staining; black arrows). e. DH case, demonstrating panoramic staining with vimentin in a subepidermal blister (red arrow), and also around dermal blood vessels and other skin appendageal structures (black arrows)(40x). $\mathbf{f}$. IHC staining with vimentin in a Senear-Usher syndrome case, demonstrating positive staining within basaloid cells of a hair follicle; note some intercellular staining within spelling, keratynocytes, as well as strong reactivity in dermal blood vessels surrounding the hair follicle (brown staining; red arrow)(400x).

\section{Discussion}

Cell migration plays a crucial role in embryonic development, wound healing, regeneration, inflammation and immune responses, as well as in dissemination of malignant neoplasms. Vimentin is often utilized as a marker of mesenchymal-derived cells, or cells undergoing an epithelial-to-mesenchymal transition (EMT) during both normal development and metastatic progression [1-4,14].

Knowing that vimentin is useful for the identification of cells of mesenchymal origin in normal and neoplastic tissues, and based on our observed tissue "compartmentalization", we suggest that rearrangement and/or sequestering of cells of mesenchymal origin occurs in the immune response in ABDs. Further, this process may contribute to the creation of blisters and other pathologic sequelae in these disorders.

In many mouse models described for ABDs, the phenomenon of redistribution of dermal mesenchymal cells has not been documented. Notably, many of these models do not exhibit a chronic $\mathrm{ABD}$ state, especially those created via injection of autoantibodies; thus, the redistribution phenomenon may represent a cellular manifestation of a chronic ABD state. Further, our findings with the reorganization of dermal tissue demonstrated by vimentin staining suggests that a complex dermal microenvironment is needed to create the immune responses in ABDs, versus a basic response previously believed to be mediated by autoantibodies and/or complement. Finally, vimentin staining may represent a useful tool to demonstrate patterns of tissue alterations secondary to the inflammatory processes in different ABDs. The pattern of vimentin staining observed is comparable to those patterns we have previously described for proteases and protease inhibitors in lesional skin in ABDs, escalating the concept that the autoimmune process extends into the dermis and below the junctional zone.

\section{REFERENCES}

1. Eriksson JE, Dechat T, Grin B, Helfand B, Mendez M, Pallari HM, et al. Introducing intermediate filaments: from discovery to disease. J Clin Invest. 2009;119:1763-71.

2. Fuchs E, Weber K. Intermediate filaments: structure, dynamics, function, and disease. Annu Rev Biochem. 1994;63:345-82.

3. Jordon RE, Kawana S, Fritz KA. Immunopathologic mechanisms in pemphigus and bullous pemphigoid. J Invest Dermatol. $1985 ; 85: 72$ s-78s.

4. Ojeh NO, Navsaria HA. An in vitro skin model to study the effect of mesenchymal stem cells in wound healing and epidermal regeneration. J Biomed Mater Res A. 2013 Sep 25. doi: 10.1002/ jbm.a.34950

5. Sams WM Jr, Gammon WR. Mechanism of lesion production in pemphigus and pemphigoid. J Am Acad Dermato. 1982;6:431-52.

6. Abreu Velez AM, Calle J, Howard MS. Autoimmune epidermal blistering diseases. Our Dermatol Online. 2013;4(Suppl.3):631-46.

7. Abreu Velez, AM, Vasquez-Hincapie DA, Howard MS. Autoimmune basement membrane and subepidermal blistering diseases. Our Dermatol Online. 2013;4(Suppl.3):647-62.

8. Abreu Velez AM, Googe PB, Howard MS. In situ immune response in skin biopsies from patients affected by autoimmune blistering diseases. Our Dermatol Online. 2013;4(Suppl.3):606-12. 
9. Abreu Velez AM, Googe PB, Howard MS. Immunohistochemistry versus immunofluoresence in the diagnosis of autoimmune blistering diseases. Our Dermatol Online. 2013;4(Suppl.3):627-30.

10. Abreu Velez AM, Yepes-Naranjo MM, Avila IC, Londoño ML, Googe PB, Velásquez-Velez JE, Velez ID, et al. Tissue inhibitor of metalloproteinase 1, Matrix metalloproteinase 9, alpha-1 antitrypsin, metallothionein and urokinase type plasminogen activator receptor in skin biopsies from patients affected by autoimmune blistering diseases. Our Dermatol Online. 2013;4:275-80.

11. Abreu Velez AM, Smith JG Jr, Howard MS. Vimentin compartmentalization in discoid lupus. N Am J Med Sci. 2010;2:10610 .
12. Abreu Velez AM, Yi H, Gao W, Smoller BR, Grossniklaus HE, Howard MS. Antibodies to pilosebaceous units along their neurovascular supply routes in a new variant of endemic pemphigus foliaceus in Colombia, South America. Eur J Dermatol. 2011;21:3715 .

13. Abreu Velez AM, Howard MS, Yi H, Gao W, Hashimoto T, Grossniklaus HE. Neural system antigens are recognized by autoantibodies from patients affected by a new variant of endemic pemphigus foliaceus in Colombia. J Clin Immunol. 2011;31:356-68. 14. Chernoivanenko IS, Minin AA, Minin AA. Role of vimentin in cell migration. Ontogenez. 2013;44:186-202.

\footnotetext{
Copyright by Ana Maria Abreu Velez, et al. This is an open access article distributed under the terms of the Creative Commons Attribution License,
} which permits unrestricted use, distribution, and reproduction in any medium, provided the original author and source are credited. 